\title{
Efecto del pH sobre el crecimiento in vitro de hongos ectomicorrícicos recolectados de plantaciones de Pinus radiata
}

\author{
Effect of $\mathrm{pH}$ on the in vitro growth of ectomycorrhizal fungi \\ collected from Pinus radiata plantations
}

\author{
Guillermo Pereira $\mathrm{C}^{\mathrm{a}^{*}}$, Jaime Herrera $\mathrm{S}^{\mathrm{a}}$, Angela Machuca $\mathrm{H}^{\mathrm{a}}$, Manuel Sánchez $\mathbf{O}^{\mathrm{b}}$ \\ *Autor de correspondencia: aUniversidad de Concepción, Departamento Forestal, Laboratorio de Biotecnología de Hongos, \\ Campus Los Angeles, J. A. Coloma 0201, Casilla 341, Los Ángeles, Chile, gpereira@udec.cl \\ bUniversidad de Concepción, Facultad de Ciencias Forestales, Departamento de Silvicultura, \\ Laboratorio de Biotecnología Forestal, Concepción, Chile.
}

\begin{abstract}
SUMMARY
In this study, the effect of the $\mathrm{pH}$ on the in vitro growth of the ectomycorrhizal fungi Rhizopogon luteolus, Suillus bellinii and Suillus luteus, collected from Pinus radiata was evaluated. The fungal cultures were grown on solid BAF (Biotin-Aneurin-Folic acid agar) medium with $\mathrm{pH}$ adjusted to $4.8,5.8,6.8$ and 7.8 and incubated for 32 days at $24 \pm 1{ }^{\circ} \mathrm{C}$. During the incubation, the radial growth rate $\left(\mathrm{mm} /\right.$ day) was determined and the area of the colonies $\left(\mathrm{cm}^{2}\right)$ and the $\mathrm{pH}$ changes of the media were also determined at the end of incubation period. The results showed that a variation of $\mathrm{pH}$ from 4.8 to 7.8 affected significantly the growth rate and the final area of colonies of $R$. luteolus and S. luteus. For R. luteolus: the highest rate of growth and colony area occurred at $\mathrm{pH}$ 5.8 and 6.8; while for S. luteus, the highest growth rate and colony area were observed at $\mathrm{pH} 4.8$ and 5.8, respectively. When the changes in the $\mathrm{pH}$ cultures at the end of incubation period were investigated, it was observed that all fungal species reduced the $\mathrm{pH}$ of the media acidifying it in a lesser or larger extent, being this effect more intense for the cultures with higher initial $\mathrm{pH}$. These results suggest that the $\mathrm{pH}$ of culture medium is determinant of the in vitro behaviour of the ectomycorrhizal fungal species. This is very important for the selection of new ectomycorrhizal species for large-scale inoculum production for application in programs of plant mycorrhization in forest nurseries.
\end{abstract}

Key words: ectomycorrhizal fungi, in vitro growth, $\mathrm{pH}$ effect, rate of radial growth.

\section{RESUMEN}

En este estudio fue evaluado el efecto del $\mathrm{pH}$ del medio de crecimiento sobre el desarrollo in vitro de tres especies de hongos ectomicorrícicos, Rhizopogon luteolus, Suillus bellinii y Suillus luteus, recolectados en plantaciones de Pinus radiata. El material fúngico fue aislado a partir de carpóforos y cultivado en medio nutritivo BAF (biotina-aneurina-ácido fólico agar) con pH ajustado a 4,8, 5,8, 6,8 y 7,8, e incubado durante 32 días a $24 \pm 1{ }^{\circ} \mathrm{C}$. En este período fue evaluada la velocidad de crecimiento radial (mm/ días) y al finalizar el ensayo fueron evaluadas el área de crecimiento de las colonias $\left(\mathrm{cm}^{2}\right)$ y la variación de $\mathrm{pH}$ del medio. Los resultados indicaron que una variación de $\mathrm{pH}$ entre 4,8 y 7,8 tiene efecto significativo sobre la velocidad y área final de crecimiento de las especies $R$. luteolus y S. luteus. Rhizopogon luteolus alcanzó los mayores crecimientos, en velocidad y área, en los valores de pH 5,8 y 6,8. Para S. luteus la mayor velocidad y área de crecimiento fueron observadas a pH 4,8 y 5,8, respectivamente. En la variación de $\mathrm{pH}$ experimentada por los medios nutritivos las tres especies disminuyeron el $\mathrm{pH}$ de los medios, siendo esto más evidente en los tratamientos de mayor $\mathrm{pH}$ inicial. Los resultados indicaron que el $\mathrm{pH}$ del medio de cultivo es determinante en el comportamiento de las especies de hongos ectomicorrícicos in vitro. Esto será de gran importancia en la selección de nuevas especies ectomicorrícicas para producir inóculo micelial en grandes cantidades para ser aplicado en programas de micorrización de plantas en viveros forestales.

Palabras clave: hongos ectomicorrícicos, cultivo in vitro, efecto del $\mathrm{pH}$, velocidad de crecimiento radial.

\section{INTRODUCCIÓN}

El pH es considerado de acuerdo con Jackson (1970) como una de las propiedades químicas más importantes del suelo, debido al significativo efecto que ejerce tanto sobre las características físicas, químicas y biológicas de éste, como también sobre el rendimiento de los cultivos (Guigon et al. 1989, Saña et al. 1996, Cepeda 1999). Esta variable puede determinar desde el punto de vista biológico el tipo de organismo que se desarrolle sobre un suelo, debido a su significativa influencia sobre la disponibilidad de nutrientes. Al respecto, Wild (1992) afirma que los 
hongos y el grupo de bacterias-actinomicetos constituyen los dos grandes grupos de microorganismos del suelo y el predominio de uno u otro grupo depende de las condiciones locales, especialmente del pH y del contenido de humedad. Entre estos microorganismos se encuentran los hongos micorrícicos, con los cuales la mayoría de las plantas vive en simbiosis (Harley y Smith 1983, Barea 1991, Honrubia et al. 1992, Brundrett et al. 1996). Esta asociación presenta múltiples beneficios para las plantas, entre los que se pueden destacar el aumento en la absorción de agua y nutrientes minerales, mayor crecimiento y supervivencia de las plantas, protección frente a infecciones de organismos patógenos y estrés ambiental (Harley y Smith 1983, Gianinazzi-Person y Azcón-Aguilar 1991, Honrubia et al. 1992). Se debe tener presente que el desarrollo y actividad de los hongos micorrícicos puede verse afectado por diversos factores del sitio, y que además cada especie fúngica (cepa o ecotipo) tiene sus propias limitaciones ecológicas, existiendo por ello algunos más benéficos que otros en determinadas condiciones ambientales. Por ello la adecuada selección de las especies de hongos micorrícicos como simbiontes y su posterior manipulación, tanto en laboratorio como en vivero, pueden ser aspectos claves para lograr con éxito el establecimiento de muchas especies vegetales en campo (Honrubia et al. 1992, Marx et al. 1994). En este intento las primeras especies a estudiar deben ser siempre aquellas que se encuentran creciendo en forma natural en los sitios de interés, más aún si ellas son consideradas pioneras en el establecimiento de algunos sistemas boscosos, como es el caso de las pertenecientes a los géneros Rhizopogon y Suillus. Dentro de este contexto, el objetivo de este estudio es analizar bajo condiciones controladas de laboratorio el efecto del $\mathrm{pH}$ del medio de cultivo sobre el crecimiento in vitro de tres especies ectomicorrícicas, comúnmente encontradas en plantaciones de Pinus radiata D. Don.

\section{MÉTODOS}

Se estudiaron los hongos ectomicorrícicos Rhizopogon luteolus Fr., Suillus bellinii (Inz.) Kuntze y Suillus luteus L. Gray, recolectados de plantaciones jóvenes de Pinus radiata $\mathrm{D}$. Don, de dos sitios de arenales de baja productividad de la provincia del Bío Bío, VIII Región, Chile. Algunas propiedades físicas y químicas de los suelos de estos sitios fueron analizadas en el Laboratorio de Suelo de la Facultad de Agronomía de la Universidad de Concepción (cuadro 1).

Estas especies fueron identificadas a través de características macroscópicas y microscópicas de sus cuerpos fructíferos (Moreno et al. 1996, Valenzuela 1998, Gerhardt et al. 2000, Lazo 2001). El material fúngico recolectado en campo fue trasladado a laboratorio, donde bajo condiciones de asepsia se diseccionaron los carpóforos extrayendo tejido ubicado inmediatamente por encima del himenio en el caso de los hongos epígeos (setas) y en la zona central
Cuadro 1. Características físicas y químicas de los suelos de los sitios de colecta.

Physical and chemical characteristics of the soils at the collections sites.

\begin{tabular}{|c|c|c|}
\hline Sitio de colecta & Especie & Características \\
\hline $\begin{array}{l}\text { Sector Paraguay } \\
37^{\circ} 12^{\prime} 20^{\prime \prime} \mathrm{S}, \\
72^{\circ} 10^{\prime} 29^{\prime \prime} \mathrm{O}\end{array}$ & $\begin{array}{l}\text { Rhizopogon } \\
\text { luteolus }\end{array}$ & $\begin{array}{l}\text { Suelo arenoso, pH 6,1; } \\
\text { MO: } 2,38 \% \text {; N: } 1,3, \mathrm{P}: \\
6,7, \mathrm{~K}: 47,9(*)\end{array}$ \\
\hline $\begin{array}{l}\text { Sector Aeródromo } \\
37^{\circ} 22^{\prime} 41^{\prime \prime} \mathrm{S} \\
72^{\circ} 25^{\prime} 30^{\prime \prime} \mathrm{O}\end{array}$ & $\begin{array}{l}\text { Suillus bellinii } \\
\text { y Suillus } \\
\text { luteus }\end{array}$ & $\begin{array}{l}\text { Suelo arenoso-francoso, } \\
\text { pH 5,6; MO: } 2,37 \% \text {; N: } \\
56,3, \mathrm{P}: 8,8, \mathrm{~K}: 119,8(*)\end{array}$ \\
\hline
\end{tabular}

MO: materia orgánica. $(*)$ Contenidos de nitrógeno, fósforo y potasio en $\mathrm{mg} / \mathrm{kg}$ de suelo seco, determinados como elementos disponibles.

de la gleba en los hongos hipógeos (Honrubia et al. 1995, Brundrett et al. 1996). Los fragmentos de tejido fueron depositados en placas de Petri con $20 \mathrm{ml}$ de medio de cultivo BAF (biotina-aneurina-ácido fólico agar) (Honrubia et al. 1995), ajustado a $\mathrm{pH}$ 5,8. Las placas fueron incubadas en oscuridad a $24 \pm 1{ }^{\circ} \mathrm{C}$, hasta obtener un crecimiento activo de los micelios (cultivos stock). Posteriormente, discos de micelio de $5 \mathrm{~mm}$ de diámetro, obtenidos de los cultivos stock fueron transferidos a nuevas placas con $20 \mathrm{ml}$ de medio nutritivo BAF, con pH ajustado a 4,8, 5,8, 6,8 y 7,8. Estas placas fueron incubadas a $24 \pm 1{ }^{\circ} \mathrm{C}$ durante 32 días, período en el cual se midió cada tres días el crecimiento radial de las colonias por el reverso de las placas con pie de metro digital. Estos datos se ajustaron mediante una ecuación de regresión lineal para calcular la pendiente de la curva de crecimiento que corresponde a la velocidad media de crecimiento de cada especie fúngica, expresada en ( $\mathrm{mm} /$ día $)$ (Santiago-Martínez et al. 1995, Vázquez-García et al. 2002). Finalizado el período de cultivo se determinó el área final de crecimiento $\left(\mathrm{cm}^{2}\right)$, graficando el perímetro de las colonias sobre papel y evaluando esta variable en un medidor de área digital (modelo LI-3100). Para cada uno de los tratamientos fue determinado el valor de $\mathrm{pH}$ final de los medios de cultivo (previa disolución en horno de microondas) utilizando un potenciómetro marca EXTECH modelo 321990, provisto de un electrodo de $\mathrm{pH}$ para medio líquido.

El estudio se estableció bajo un diseño completamente aleatorio, considerando tres repeticiones por tratamiento (Montgomery 1991). Los resultados obtenidos fueron evaluados a través de análisis de varianza y cuando hubo diferencias significativas entre tratamientos, éstas se identificaron a través de la prueba de Tukey para comparaciones múltiples (Steel y Torrie 1989).

\section{RESULTADOS}

Velocidad media de crecimiento. Los resultados muestran que la variación de $\mathrm{pH}$ del medio tuvo efecto sobre la velocidad 
de crecimiento de $R$. luteolus y S. luteus (figura 1). Se observa que $R$. luteolus presentó las mayores velocidades medias de crecimiento, independiente del rango de $\mathrm{pH}$ estudiado. La máxima velocidad de crecimiento para esta especie se encontró en los tratamientos a pH 5,8 y $6,8(1,98$ y $1,79 \mathrm{~mm} /$ día, respectivamente), resultados que alcanzaron significancia estadística al ser comparados con los tratamientos a $\mathrm{pH}$ 4,8 y 7,8 (1,34 y $1,09 \mathrm{~mm} / \mathrm{dí}$, respectivamente), donde se lograron los menores crecimientos.

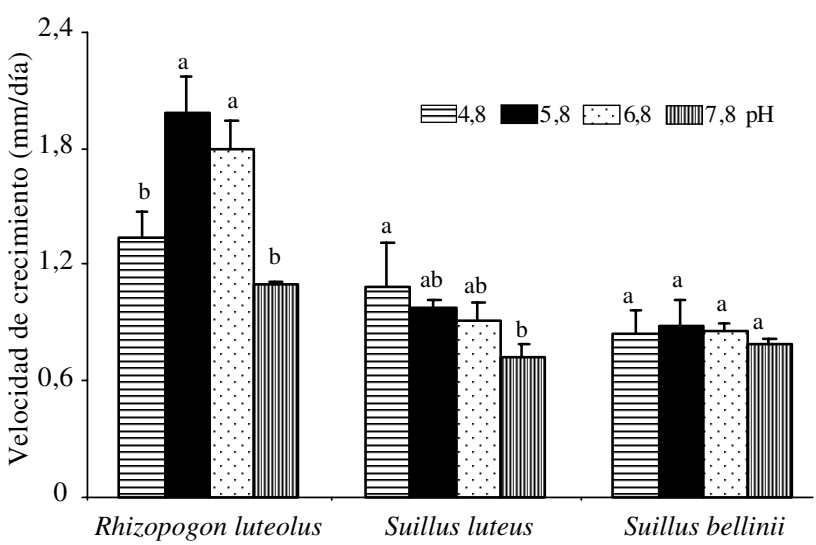

Figura 1. Efecto del pH del medio de cultivo en la velocidad de crecimiento (mm/día) de las especies de hongos ectomicorrícicos. Para una misma especie, letras diferentes sobre las barras indican que existen diferencias significativas entre los tratamientos (Tukey, $P<0,05)$. Los datos representan la media $\pm \mathrm{S}$ de tres réplicas por tratamiento.

Effect of the $\mathrm{pH}$ on the rate of growth (mm/day) of ectomycorrhizal fungi. For the same fungal species, bars with different letters indicate values with significant differences (Tukey, $P<0.05$ ). The values represent means of three replicates \pm S.E.

Suillus luteus fue la segunda especie con mayor crecimiento, logrando su máxima velocidad en el tratamiento a $\mathrm{pH} 4,8(1,08 \mathrm{~mm} / \mathrm{día})$, velocidades que decrecieron al aumentar el $\mathrm{pH}$ del medio (figura 1), produciéndose diferencias significativas entre el crecimiento de las colonias cultivadas en medio nutritivo a $\mathrm{pH} 4,8$ respecto de las colonias cultivadas a pH 7,8 $(0,72 \mathrm{~mm} /$ día $)$. Se observó que $S$. bellinii presentó la menor velocidad de crecimiento, superando sólo a S. luteus en el tratamiento a pH 7,8. Las velocidades de crecimiento de esta especie no presentaron diferencias estadísticas, independientemente de los valores de $\mathrm{pH}$ ensayados.

Área media de crecimiento. El área de crecimiento de las tres especies ectomicorrícicas también se vio afectada por la variabilidad del $\mathrm{pH}$ del medio (figura 2 ). Se observa que $R$. luteolus fue la especie con mayor área de crecimiento en todas las condiciones de $\mathrm{pH}$ estudiadas, alcanzando un área de crecimiento significativamente mayor en los cultivos a pH 5,8 y $6,8\left(42,7\right.$ y $\left.33,2 \mathrm{~cm}^{2}\right)$, respecto a los obtenidos a $\mathrm{pH} 4,8$ y 7,8 .
Por otra parte, se observó que la especie S. luteus logró sus máximos crecimientos en área total en los cultivos a pH 5,8 y $6,8\left(17,0\right.$ y $11,9 \mathrm{~cm}^{2}$, respectivamente), disminuyendo a medida que aumentó o bajó el valor del $\mathrm{pH}$, existiendo diferencias significativas entre el cultivo a pH 5,8 , respecto de las colonias cultivadas a $\mathrm{pH} 4,8$ y 7,8 (figura 2).

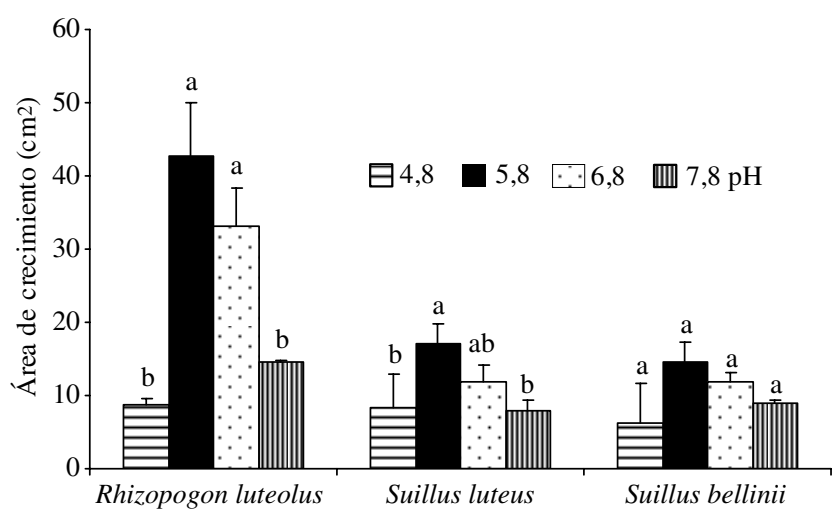

Figura 2. Efecto del $\mathrm{pH}$ del medio de cultivo en el área de crecimiento $\left(\mathrm{cm}^{2}\right)$ de las especies de hongos ectomicorrícicos. Para una misma especie, letras diferentes sobre las barras indican que existen diferencias significativas entre los tratamientos (Tukey, $P<0,05)$. Los datos representan la media $\pm \mathrm{S}$ de tres réplicas por tratamiento.

Effect of the $\mathrm{pH}$ on the area of growth $\left(\mathrm{cm}^{2}\right)$ of ectomycorrhizal fungi. For a same fungal species, the same letters on the bars are not significantly different $(P<0,05)$. The values represent means of three replicates \pm S.E.

La especie $S$. bellinii tuvo un comportamiento similar a $S$. luteus, logrando su mayor área total de crecimiento a pH 5,8 $\left(14,5 \mathrm{~cm}^{2}\right)$, mientras que los menores valores se obtuvieron a $4,8\left(6,2 \mathrm{~cm}^{2}\right)$ y $\mathrm{pH} 7,8\left(8,9 \mathrm{~cm}^{2}\right)$. Sin embargo, en este caso las diferencias observadas no fueron estadísticamente significativas.

Variación de $\mathrm{pH}$ del medio de cultivo. Los resultados indican que las tres especies de hongos ectomicorrícicos provocaron cambios en el $\mathrm{pH}$ del medio de crecimiento, respecto al $\mathrm{pH}$ inicial, tendiendo siempre hacia la acidificación (cuadro 2).

Se observó que el grado de acidificación aumentó a medida que el valor de $\mathrm{pH}$ inicial del medio fue mayor (cuadro 2). De las tres especies ectomicorrícicas, S. bellinii fue la que más acidificó el medio en todos los tratamientos, experimentando cambios de 0,51 unidades de $\mathrm{pH}$ en las colonias cultivadas a pH 4,8, alcanzando cambios de hasta 2,76 unidades para aquellas cultivadas a $\mathrm{pH} 7,8$. Por otro lado, S. luteus fue la especie que menos modificó el pH del medio de crecimiento respecto al inicial, excepto en el tratamiento a $\mathrm{pH} 4,8$. 
Cuadro 2. Modificación del pH del medio de cultivo durante el crecimiento in vitro de $R$. luteolus, S. bellinii y $S$. luteus.

$\mathrm{pH}$ modification of the culture medium during the in vitro growth of R. luteolus, S. bellinii and S. luteus.

\begin{tabular}{lcccr}
\hline & \multicolumn{5}{c}{$\mathrm{pH}$ inicial del medio de cultivo } \\
\cline { 2 - 5 } Especies & 4,8 & 5,8 & 6,8 & 7,8 \\
\cline { 2 - 5 } & \multicolumn{5}{c}{$\mathrm{pH}$ final } \\
\cline { 2 - 5 } & 4,59 & 4,58 & 4,68 & 5,61 \\
Rhizopogon luteolus & 4,29 & 4,14 & 4,48 & 5,04 \\
Suillus bellinii & 4,49 & 4,99 & 5,42 & 6,46 \\
Suillus luteus & &
\end{tabular}

\section{DISCUSIÓN}

El crecimiento in vitro de las especies de hongos ectomicorrícicos respondió de forma diferente a los distintos valores de $\mathrm{pH}$ inicial del medio de cultivo ensayados. De las tres especies, $R$. luteolus fue la que presentó los mayores crecimientos, alcanzándose los máximos valores tanto en velocidad como en área en los pH 5,8 y 6,8. Al respecto Vázquez-García et al. (2002), en un estudio similar con cepas de Rhizopogon sp., encontraron los óptimos de crecimiento en $\mathrm{pH} 6,0$, con velocidades medias $(1,28$ $\mathrm{mm} / \mathrm{día}$ ) inferiores a las obtenidas en este estudio. Por otra parte, estudios realizados por Torres y Honrubia (1991), con seis especies de hongos ectomicorrícicos recolectados de bosques de Pinus sp., mostraron que R. luteolus y $R$. roseolus presentaron sus máximos de crecimiento en valores de $\mathrm{pH}$ mayores a 7,2.

Los resultados encontrados muestran que, en general, las especies R. luteolus y $S$. luteus, tienden a crecer mejor en condiciones de acidez que de alcalinidad, siendo el tratamiento a pH 7,8 el que más afectó la velocidad media de crecimiento (figura 1), resultados que concuerdan con los estudios de Hung y Trappe (1983), Willenborg et al. (1990) y Hormilla (1995), en el sentido de que los hongos ectomicorrícicos tienen una naturaleza acidófila cuando crecen en condiciones de cultivos puros.

Por otra parte, al analizar los valores de $\mathrm{pH}$ donde se alcanzaron los mayores crecimientos de las tres especies de hongos ectomicorrícicos (figuras 1 y 2) se observa que, en general, el mejor comportamiento que presentan los hongos cultivados in vitro se produce en medios nutritivos cuyo $\mathrm{pH}$ es similar a aquel registrado en los suelos en donde éstos estaban creciendo en forma natural (cuadro 1). Ello indica que las condiciones de $\mathrm{pH}$ del sector de colecta del material fúngico deben ser consideradas para optimizar el cultivo y propagación de los hongos en laboratorio y, por ende, para mejorar la micorrización en la producción de plantas en vivero.

La tendencia general de las especies estudiadas es acidificar los medios de crecimiento (cuadro 2), siendo S. bellinii la que más acidificó los medios y S. luteus la que menos cambios produjo. Se debe destacar que ambas especies provenían del mismo sitio de colecta, por lo cual esta diferencia en comportamiento pudiese estar relacionada con las estrategias propias de cada especie para aumentar la biodisponibilidad de nutrientes. De acuerdo con algunos autores (Hung y Trappe 1983, García-Rodríguez et al. 2006) durante el desarrollo del micelio in vitro de hongos ectomicorrícicos se producen una serie de ácidos orgánicos, además de la absorción de iones, lo que provocaría la acidificación del medio de cultivo. Ello sugiere que debería existir una correlación entre un mayor crecimiento de la especie (velocidad y/o área) y una mayor disminución de $\mathrm{pH}$ del medio, lo cual no se observa en los resultados obtenidos en este estudio. Al respecto Sánchez (1997) y Sánchez et al. (2001), estudiando especies similares, no consiguieron relacionar la mayor producción de biomasa con un mayor descenso del $\mathrm{pH}$ del medio.

Los resultados encontrados en este estudio sugieren que en la búsqueda de la optimización planta-hongo-sitio, para algunas especies de hongos ectomicorrícicos, se debe tener en consideración la variable $\mathrm{pH}$ del medio de crecimiento, pues ello permitirá al silvicultor optimizar el crecimiento de las especies fúngicas en laboratorio, mejorar la micorrización de las plantas en vivero, así como también elegir el sitio más adecuado para que el hongo micorrícico con su respectiva planta huésped expresen el máximo potencial de desarrollo. En este sentido se hace necesario aumentar los conocimientos de las especies micorrícicas que están creciendo en bosques chilenos, y de esta forma poder seleccionar para las micorrizaciones en viveros aquellas que sean más idóneas para potenciar el crecimiento en campo. A su vez, en laboratorio se hace necesario estudiar metodologías y condiciones de cultivo ( $\mathrm{pH}$, temperatura y composición de los medios) más adecuadas para cada especie de interés, y así poder conseguir en periodos cortos de tiempo producciones masivas de inóculos que puedan ser utilizadas a escala operacional en los viveros forestales. Se debe destacar que las tres especies en estudio, pertenecientes a los géneros Rhizopogon y Suillus, son recurrentes en los bosques de Pinus radiata en Chile, presentándose con mayor o menor abundancia dependiendo de las condiciones edafoclimáticas de los sitios. Estas especies son consideradas pioneras en el establecimiento de algunos sistemas boscosos, condición que les proporciona un alto potencial para ser consideradas en micorrizaciones controladas.

\section{CONCLUSIONES}

El pH del medio de cultivo tiene efectos significativos sobre el crecimiento in vitro del micelio vegetativo de $R$. luteolus y S. luteus.

De las tres especies de hongos ectomicorrícicos, $R$. luteolus presentó los mayores crecimientos, independiente de los tratamientos probados, indicando el alto 
potencial que presenta esta especie para ser usada en programas de inoculación bajo diferentes condiciones de $\mathrm{pH}$ en los suelos.

En general, los valores de $\mathrm{pH}$ del medio de cultivo donde se observaron los mayores crecimientos se correlacionan con los valores de $\mathrm{pH}$ de los suelos, donde los hongos crecían en forma natural.

El crecimiento de $R$. luteolus, $S$. bellinii y $S$. luteus disminuyó el pH del medio de cultivo, siendo este efecto más evidente en los cultivos donde el $\mathrm{pH}$ inicial fue de 7,8 .

\section{AGRADECIMIENTOS}

Los autores agradecen el financiamiento otorgado a través del FONDECYT 1030620 y a la Dirección de Investigación de la Universidad de Concepción DIUC 204.415.005-1.0.

\section{REFERENCIAS}

Barea JM. 1991. Vesicular-Arbuscular mycorrhizae as modifiers of soil fertility. Advances in Soil Science 15:1-40.

Brundrett M, N Bougher, T Grove, N Malajczuk. 1996. Working with mycorrhizas in forestry and agriculture. Canberra. ACIAR. 374 p.

Cepeda J. 1999. Química de suelos. México. Editorial Trillas. $167 \mathrm{p}$.

García-Rodríguez JL, J Pérez-Moreno, A Aldrete, V CetinaAlcalá, H Vaquera-Huerta. 2006. Caracterización del hongo silvestre ectomicorrícico Pisolithus tinctorius (Pers.) Coke et Couch en cultivo y en simbiosis con eucalipto y pino. Agrociencia 40:665-676.

Gerhardt E, J Vila, X Llimona. 2000. Hongos de España y de Europa. Barcelona, España. Omega. 957 p.

Gianinazzi-Person V, C Azcón-Aguilar. 1991. Fisiología de las micorrizas vesículo-arbusculares. In Olivares J, JM Barea eds. Fijación y movilización biológica de nutrientes. CSIC. II. Granada, España. p. 175-201.

Guigon B, B Thonnelier, B Duzan, B Felix-Faure. 1989. Pour valoriser les analyses de sol. Purpar 134:3-88.

Harley JL, E Smith. 1983. Mycorrhizal symbiosis. London, UK. Academic Press. 483 p.

Honrubia M, P Torres, G Díaz, A Cano. 1992. Manual para micorrizar plantas en viveros forestales. Ministerio de Agricultura, Pesca y Alimentación. Madrid, España. ICONA. 47 p.

Honrubia M, P Torres, G Díaz, A Morte. 1995. Biotecnología Forestal: Técnicas de micorrización y micropropagación de plantas. Murcia, España. 84 p.
Hormilla S. 1995. Estudio de la simbiosis ectomicorrícica en Quercus robur L. y valoración de su influencia en la fisiología de la planta. Tesis Doctoral. Universidad del País Vasco. 279 p.

Hung L, J M Trappe. 1983. Growth variation between and within species of ectomycorrhizal fungi in response to $\mathrm{pH}$ in vitro. Mycologia 75:234-241.

Jackson M L. 1970. Análisis químico de suelos. Barcelona, España. Omega. 662 p.

Lazo W. 2001. Hongos de Chile. Atlas micológico. Santiago, Chile. 223 p.

Marx D H, J L. Ruehle, D E. Cordell. 1994. Methods for studying nursery and field response of trees to specific ectomycorrhiza. In Techniques for Mycorrhizal Research: Methods in Microbiology. Norris JR, D Read, AK Varna eds. London, UK. Academic Press. p. 383-411.

Montgomery D. 1991. Diseño y análisis de experimentos. México DF, México. Grupo Editorial Iberoamérica. 149 p.

Moreno B, F Jiménez, J Gómez, F Infante. 1996. Setas de Andalucía. Sevilla, España. 376 p.

Sánchez F. 1997. Hongos ectomicorrícicos del Maestrazgo: Estudio taxonómico, ecológico y fisiológico. Tesis Doctoral. Universidad de Murcia. 397 p.

Sánchez F, M Honrubia, P Torres. 2001. Effects of pH, water stress and temperature on in vitro culture of ectomicorrhizal fungi from Mediterranean forests. Cryptogamie Mycologie 22:243-258.

Saña J, J More, A Cohi. 1996. La gestión de la fertilidad de los suelos. Ministerio de Agricultura, Pesca y Alimentación. Madrid, España. 277 p.

Santiago-Martínez G, L Varela, A Estrada-Torres, V Cuaxilo. 1995. Efecto de seis medios de cultivo sobre el crecimiento de tres cepas de Pisolithus tinctorius. Revista Mexicana de Micología 11:57-68.

Steel R, J Torrie. 1989. Bioestadística: Principios y procedimientos. México DF, México. $2^{\mathrm{a}}$ ed. McGraw Hill. 662 p.

Torres P, M Honrubia. 1991. Dinámica de crecimiento y caracterización de algunos hongos ectomicorrícicos en cultivo. Cryptogamie Mycologie 12:183-192.

Vázquez-García A, G Santiago-Martínez, A Estrada-Torres. 2002. Influencia del $\mathrm{pH}$ en el crecimiento de quince cepas de hongos ectomicorrizógenos. Anales del Instituto de Biología, Universidad Nacional Autónoma de México. Serie Botánica 73:1-15.

Valenzuela E. 1998. Guía de campo para setas (Agaricales) de la Isla Teja, Valdivia. Valdivia, Chile. 50 p.

Wild A. 1992. La población microbiana del suelo. In Condiciones del suelo y desarrollo de las plantas según Russell. Madrid, España. Ediciones Mundi-Prensa. p. 471-494

Willenborg A, D Schmitz, J Lelley. 1990. Effects of environmental stress factors on ectomycorrhizal fungi in vitro. Canadian Journal of Botany 68:1741-1746. 